\title{
Exosomes derived from mature chondrocytes facilitate subcutaneous stable ectopic chondrogenesis of cartilage progenitor cells
}

Yahong Chen ${ }^{1}$, Ke Xue ${ }^{1}$, Xiaodie Zhang ${ }^{1}$, Zhiwei Zheng ${ }^{2,3,4^{*}}$ and Kai Liu ${ }^{1 *}$

\begin{abstract}
Background: Developing cartilage constructed with the appropriate matrix composition and persistent chondrogenesis remains an enduring challenge in cartilage defects. Cartilage progenitor cell (CPC)-based tissue engineering has attracted recent attention because of its strong chondrogenic differentiation capacity. However, due to the lack of a suitable chondrogenic niche, the clinical application of CPC-regenerated cartilage in the subcutaneous environment remains a challenge. In this study, exosomes derived from chondrocytes (CC-ExOs) were used to provide the CPC constructs with a cartilage signal in subcutaneous environments for efficient ectopic cartilage regeneration.

Methods: Rabbit CPC-alginate constructs were prepared and implanted subcutaneously in nude mice. CC-Exos were injected into the constructs at the same dose (30 $\mu \mathrm{g}$ exosomes per $100 \mu \mathrm{L}$ injection) after surgery and thereafter weekly for a period of 12 weeks. Exosomes derived from bone mesenchymal stem cells (BMSC-Exos) were used as the positive control. The mice in the negative control were administered with the same volume of PBS. At 4 and 12 weeks after implantation, the potential of CC-Exos and BMSC-Exos to promote chondrogenesis and stability of cartilage tissue in a subcutaneous environment were analyzed by histology, immunostaining, and protein analysis. The influences of BMSCExos and CC-Exos on chondrogenesis and angiogenic characteristics in vitro were assessed via coculturing with CPCs and human umbilical vein endothelial cells.
\end{abstract}

Results: The CC-Exos injection increased collagen deposition and minimized vascular ingrowth in engineered constructs, which efficiently and reproducibly developed into cartilage. The generated cartilage was phenotypically stable with minimal hypertrophy and vessel ingrowth up to 12 weeks, while the cartilage formed with BMSC-Exos was characterized by hypertrophic differentiation accompanied by vascular ingrowth. In vitro experiments indicated that CC-Exos stimulated CPCs proliferation and increased expression of chondrogenesis markers while inhibiting angiogenesis.

Conclusions: These findings suggest that the novel CC-Exos provides the preferable niche in directing stable ectopic chondrogenesis of CPCs. The use of CC-Exos may represent an off-the-shelf and cell-free therapeutic approach for promoting cartilage regeneration in the subcutaneous environment.

Keywords: Cartilage, Progenitor/stem cells, Exosomes, Chondrocytes, Bone mesenchymal stem cells, Chondrogenesis,

\footnotetext{
* Correspondence: surgeonzheng@sjtu.edu.cn; drkailiu@126.com

2Department of Oral and Maxillofacial-Head and Neck Oncology, Shanghai

Ninth People's Hospital, College of Stomatology, Shanghai Jiao Tong

University School of Medicine, 639 ZhiZaoJu Road, Shanghai 200011,

People's Republic of China

'Department of Plastic and Reconstructive Surgery, Shanghai Ninth People's

Hospital, College of Stomatology, Shanghai Jiao Tong University School of

Medicine, 639 ZhiZaoJu Road, Shanghai 200011, People's Republic of China

Full list of author information is available at the end of the article
}

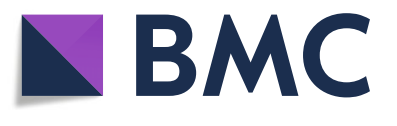

(อ) The Author(s). 2018 Open Access This article is distributed under the terms of the Creative Commons Attribution 4.0 International License (http://creativecommons.org/licenses/by/4.0/), which permits unrestricted use, distribution, and

reproduction in any medium, provided you give appropriate credit to the original author(s) and the source, provide a link to the Creative Commons license, and indicate if changes were made. The Creative Commons Public Domain Dedication waiver (http://creativecommons.org/publicdomain/zero/1.0/) applies to the data made available in this article, unless otherwise stated. 


\section{Background}

The structural and functional repair of sizeable subcutaneous cartilage defects remains a challenge in plastic and reconstructive surgery $[1,2]$. Strategies for cartilage defects include autologous chondrocytes implantation and matrix-assisted chondrocyte implantation [3, 4]. While successful in some respects, each of these therapies has limitations, including donor limitation, donor morbidity, and degradation of the graft tissue [5, 6]. In recent years, stem cell-based cartilage tissue engineering has shown great promise [7]. Cartilage progenitor/stem cells (CPCs), which are considered an attractive cell source, have been increasingly investigated in cartilage regeneration because of their strong chondrogenic potential [8-10]. Unfortunately, the lack of a suitable chondrogenic niche still hinders efficient and stable ectopic chondrogenesis of progenitor cells in subcutaneous environments [11, 12].

Indeed, the tissue regeneration niche plays a crucial role in determining the ultimate phenotype of implanted stem cells [13-15]. Chondrocytes are one of the major niche cell types in cartilage and play an essential role in the maintenance of the cartilage microenvironment. Many studies have demonstrated the improved chondrogenesis of stem cells after coculturing with chondrocytes $[1,16]$. Chondrocytes can also create a proper chondrogenic niche for stabilizing the chondrogenic phenotype of stem cells in ectopic cartilage regeneration [17]. Furthermore, it is hypothesized that the chondrocytes empower the chondrogenic efficacy of progenitor cells mainly through the paracrine effects of trophic factors $[16,18,19]$.

Among the numerous factors secreted, exosomes have been identified as the principal agent in mediating the therapeutic efficacy of endogenous or grafted cells in several disease indications [20,21]. Exosomes are nanosized (30-200 nm), bi-lipid membrane vesicles secreted by most cell types. Exosomes have been found to contain various types of bioactive microRNAs, nucleic acids, proteins, and unique gene products [22-24]. Additionally, it was reported that exosomes could transfer trophic factors to neighboring cells and serve as mediators of intercellular communication [25]. In contrast to the conventional cell-based therapy, the use of exosomes is advantageous from the perspectives of off-the-shelf and cell-free regenerative medicine approach and the ease of minimally invasive injection.

However, it is still unknown whether chondrogenesis can be enhanced by exosomes derived from chondrocytes (CC-Exos). Moreover, the interaction between CC-Exos and cartilage hypertrophy has not been elucidated. Here, the central hypothesis that CC-Exos promotes cartilage regeneration and stabilizes the cartilage tissue phenotype via the inhibition of angiogenesis is tested using a CPC-based subcutaneous cartilage regeneration model. In addition, the exosomes derived from bone mesenchymal stem cells (BMSC-Exos) have now been used for in situ cartilage defect repair with robust cartilage tissue formation [21, 26]. BMSC-Exos were recently reported to accelerate neo-tissue filling and enhance matrix synthesis of type II collagen and sulfated glycosaminoglycan [27-30]. In this study, BMSC-Exos were used as the positive control to test the null hypothesis that there is no difference between CC-Exos and BMSC-Exos in cartilage regeneration.

\section{Methods}

Isolation and culture of CPCs, BMSCs, and chondrocytes

$\mathrm{CPCs}$ in the auricular cartilage tissue of rabbits (provided by Shanghai Chuansha Breeding Factory, $n=6$ ) were harvested via differential adhesion to fibronectin as described previously [31, 32]. Briefly, cells were seeded onto 100-mm plastic petri dishes (pretreated with $10 \mu \mathrm{g} /$ $\mathrm{mL}$ fibronectin overnight at $37^{\circ} \mathrm{C}$ ) in low-glucose Dulbecco's modified Eagle's medium (DMEM, Gibco, USA). After $20 \mathrm{~min}$, the cells were rinsed twice with phosphate-buffered saline (PBS) and cultured in low-glucose DMEM containing 10\% fetal bovine serum (FBS, Gibco, USA). BMSCs and chondrocytes from rabbits were isolated and expanded according to previously established methods [31,33]. The isolated cells were cultured in DMEM supplemented with 10\% FBS and expanded to passage 2 (P2) to extract exosomes.

\section{Preparation of CC-Exos and BMSC-Exos}

After reaching $80 \%$ confluency, chondrocytes and BMSCs were rinsed with PBS and cultured with serum-free medium for $48 \mathrm{~h}$. CC-Exos and BMSC-Exos were both isolated and purified from the conditioned medium following a previous protocol [34]. In detail, the obtained conditioned medium was centrifuged at $3000 \times g$ for $30 \mathrm{~min}$ at $4{ }^{\circ} \mathrm{C}$, followed by filtering with a $0.45-\mu \mathrm{m}$ and a $0.22-\mu \mathrm{m}$ filter (SteritopTM, Millipore, USA) to remove the remaining cells and cellular debris. Finally, exosomes were isolated by size fractionation and concentrated $50 \times$ by centrifugation using an Ultra-clear tube (Millipore) with a molecular weight cutoff of $100 \mathrm{kDa}$. Exosomes were stored at $-80{ }^{\circ} \mathrm{C}$ for the following experiments. Nano-Sight (NS300, Malvern, England), transmission electron microscopy (TEM, JEOL microscope, JSM-7001TA, Tokyo, Japan), and Western blot were used to identify exosomes.

\section{Exosome labeling and exosome uptake studies}

Isolated CC-Exos or BMSC-Exos were labeled with CM-Dil red fluorescent membrane linker dye (Invitrogen, Waltham, MA, USA) as previously described [35, 36]. Briefly, $1 \mu \mathrm{M}$ cell-labeling solution was added to $200 \mu \mathrm{g}$ exosomes suspended in $1 \mathrm{~mL}$ PBS and was incubated for 
5 min at $37{ }^{\circ} \mathrm{C}$ and $15 \mathrm{~min}$ at $4{ }^{\circ} \mathrm{C}$. Subsequently, the mixture was washed to remove unbound CM-Dil. CPCs were incubated with CM-Dil-labeled exosomes $(30 \mu \mathrm{g} / \mathrm{mL})$ for $12 \mathrm{~h}$ according to a previous study [21]. Then, cells were washed twice with PBS, fixed in $4 \%$ paraformaldehyde, and stained with phalloidin and DAPI. Finally, cells were observed under a Zeiss Confocal LSM 710 microscope (Carl Zeiss, Jena, Germany) to determine the uptake of the labeled exosomes.

\section{In vivo chondrogenesis of CPCs induced by exosome in subcutaneous non-chondrogenic sites}

All procedures were approved by the Animal Research Committee of Shanghai Jiao Tong University Affiliated Ninth People's Hospital. Implants were formed by encapsulating 1 million CPCs in $100 \mu \mathrm{L} 1.5 \%$ (wt/vol) sodium alginate (Aladdin, China) using $100 \mathrm{mM} \mathrm{CaCl}_{2}$ [37]. The engineered tissues were implanted subcutaneously as previously reported $[17,37]$ in 30 female nude mice; each mouse was randomly assigned to receive a local injection of PBS, CC-Exos, or BMSC-Exos. Exos solutions in PBS were prepared under sterile conditions. CC-Exos and BMSC-Exos (30 $\mu$ g exosomes per $100 \mu \mathrm{L}$ injection) were administered subsequently on a weekly basis [38, 39]. Five injection sites evenly distributed in the construct were determined, and $20-\mu \mathrm{L}$ solution was injected per site. The same volume of PBS was used as the negative control. The detailed process is shown in Scheme 1.

\section{Histology}

At 4 weeks or 12 weeks, the samples were explanted and histologically analyzed. After gross observation, samples were fixed in $4 \%$ paraformaldehyde for $24 \mathrm{~h}$. The samples were dehydrated with a graded alcohol series, embedded in paraffin, and sectioned perpendicularly to the implants into 5- $\mu$ m-thick sections. Hematoxylin and eosin $(H \& E)$, safranin-O/fast green (S-F), and toluidine blue (T-B) were used for histological observations.

\section{Immunohistochemistry and immunofluorescence}

Immunohistochemistry was performed as previously described [12, 27]. Dewaxed sections were washed in PBS, and endogenous peroxidase activity was quenched by immersion in 2\% $(v / v)$ hydrogen peroxide for $5 \mathrm{~min}$. Antigen retrieval was carried out by incubating the sections with a sodium citrate buffer for $30 \mathrm{~min}$. After additional washes in PBS, the sections were blocked with $1.5 \%$ goat serum for $30 \mathrm{~min}$ at room temperature followed by incubation with the primary antibody (COL II or COL X Abcam, Cambridge, UK) overnight at $4{ }^{\circ} \mathrm{C}$. The sections were then incubated with a peroxidase-conjugated secondary antibody, visualized with a 3,3-diaminobenzidine solution (DAB Substrate Kit, Burlingame, CA, USA), and counter-stained with hematoxylin.

For immunofluorescence examination, the sections were incubated with primary antibodies (anti-CD31, Abcam) overnight at $4{ }^{\circ} \mathrm{C}$. An Alexa Fluor 594-labeled secondary antibody was applied under light protection. The nuclei were counter-stained with $0.1 \mathrm{mg} / \mathrm{mL}$ DAPI, and the stained sections were examined using a Zeiss Confocal LSM 710 microscope (Carl Zeiss, Jena, Germany).

\section{Western blot}

The protocol and procedure for Western blot were performed as described in previous reports [40]. For each sample, the $30 \mu$ g extracted total protein was loaded onto a 1015\% SDS/PAGE gel. The gel-separated protein was then transferred to a PVDF membrane (Millipore) and incubated

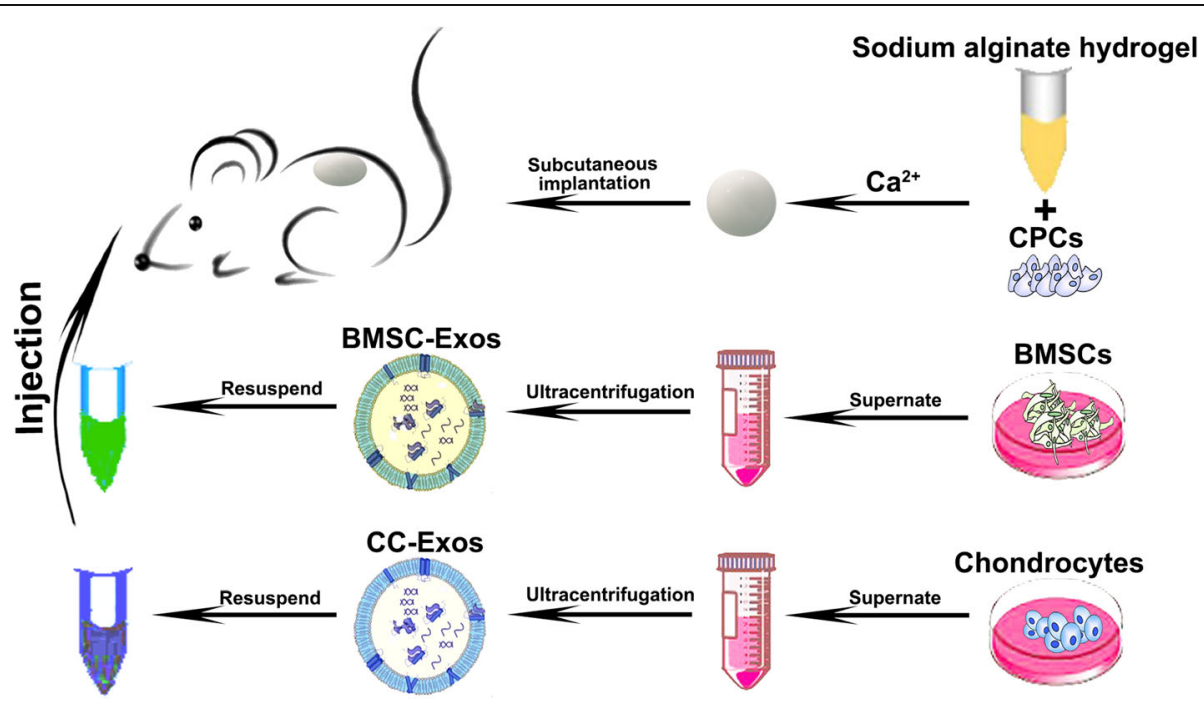

Scheme 1 Schematic illustration of the preparation of exosomes and their applications in vivo 
with the primary antibodies of anti-transforming growth factor- $\beta$ (TGF- $\beta, \quad$ Abcam), anti-SMAD2/3 (Abcam), anti-collagen type II (COL II, Abcam), anti-SOX-9 (Abcam), anti-collagen type $\mathrm{X}$ (COL X, Abcam), anti-Indian hedgehog (IHH, Affinity, OH, USA), anti-matrix metalloproteinase 13 (MMP 13, Affinity), anti-stem cell-derived factor 1 (SDF-1, Affinity), anti-vascular endothelial growth factor (VEGF, Affinity), and anti- $\beta$-actin (Invitrogen) at $37{ }^{\circ} \mathrm{C}$ for $2 \mathrm{~h}$, followed by incubation with horseradish peroxidase-conjugated secondary antibodies. Protein expression was visualized, and the values were normalized against $\beta$-actin.

\section{Cell proliferation}

The various effects of exosomes on the proliferation of CPCs were evaluated using a Cell Counting Kit-8 (CCK8, Dojindo Laboratories, Kumamoto, Japan). CPCs pretreated with $10 \mu \mathrm{g} / \mathrm{mL}$ or $30 \mu \mathrm{g} / \mathrm{mL}$ of the two different exosomes were seeded into 96-well plates, the media were changed every other day for 7 days, and cell proliferation curves were constructed by measuring with a microplate reader at a wavelength of $450 \mathrm{~nm}$.

Ki67 staining was used to determine the effect of exosomes on CPC proliferation. CPCs were incubated with CM-Dil-labeled exosomes $(10 \mu \mathrm{g} / \mathrm{mL}$ or $30 \mu \mathrm{g} / \mathrm{mL})$ for $12 \mathrm{~h}$, and the cells cultured without exosomes were used as the negative control. Then, cells were fixed with $4 \%$ formaldehyde for $30 \mathrm{~min}$ at room temperature, washed twice with PBS, and permeabilized with ice-cold methanol for $5 \mathrm{~min}$ at $4{ }^{\circ} \mathrm{C}$. Blocking was performed with $5 \%$ goat serum for $30 \mathrm{~min}$ at $37{ }^{\circ} \mathrm{C}$, followed by incubation with anti-Ki67 at a concentration of 1:1000 (Abcam) overnight at $4{ }^{\circ} \mathrm{C}$, then washed twice with PBS. Incubation with anti-rabbit fluorescent-conjugated secondary antibody for $1 \mathrm{~h}$ at room temperature in the dark was then performed. CPCs were washed twice with PBS and stained with DAPI. Ki67, CM-Dil, and DAPI immunofluorescence images were captured using a confocal microscope.

\section{Quantitative real-time polymerase chain reaction}

Total RNA was isolated and reverse transcription for cDNA synthesis was performed as previously described [21, 41]. Quantitative real-time PCR (qRT-PCR) was performed using a Power SYBR Green PCR Master Mix (Applied Biosystems) in a real-time thermal cycler (Stratagene, La Jolla, CA, USA). Glyceraldehyde 3-phosphate dehydrogenase (GADPH) was used as an internal control gene to normalize the expression of the other mRNAs. The results obtained after calibration with the GADPH expression level were calculated using the $2^{-\Delta \Delta C T}$ method and presented as fold increases relative to the negative control. The primers for qRT-PCR analysis are listed in Table 1.
Table 1 Primers used for real-time RT-PCR

\begin{tabular}{ll}
\hline Genes & Sequence (5'-3') \\
\hline COL II & F: TCC TGT GCG ACG ACA TAA TCT; R: GCA GTG GCG AGG TCA \\
& GTA G \\
SOX-9 & F: AGG TGC TCA AGG GCT ACG AC; R: TTG ACG TGG GGC TTG \\
& TTC T \\
VEGF & F: CCT TTG TGG TGG ACG CTA TC; R: CCG AAG TGA CTT GGG \\
& AAC TTG \\
SDF-1 & F: ATC CTC AAC ACG CCC AAC TG; R: TGA CCC GCC TCT CAC \\
& ATC TT \\
GAPDH & F: ATG GTG AAG GTC GGA GTG A; R: AAC ATC CAC TTT GCC \\
& AGA GTT A \\
\hline
\end{tabular}

\section{In vitro migration assay}

The migration effects of CPCs with various exosomes was evaluated using a transwell assay [42, 43]. CPCs were placed in the upper chamber of transwell inserts (Corning, NY, USA). Different culture medium was added in the lower chamber according to the group designation: negative control (NC)-DMEM medium with 1\% FBS; positive control (PC)-DMEM medium with 10\% FBS; CC-Exos group (CC-Exos)-DMEM medium with $1 \% \mathrm{FBS}$ and $30 \mu \mathrm{g} / \mathrm{mL}$ CC-Exos; BMSC-Exos group (BMSC-Exos)-DMEM with $1 \%$ FBS and $30 \mu \mathrm{g} / \mathrm{mL}$ BMSC-Exos. After incubating at $37^{\circ} \mathrm{C}$ for $6 \mathrm{~h}$ or $12 \mathrm{~h}$, cells on the lower side of the insert filter were stained with phalloidin for F-actin and DAPI for nuclei, and the numbers of cells were counted. Migration of CPCs cultured with various exosomes was further evaluated using a scratch wound assay as described previously [23]. The cells that migrated from the original wound edge at $0 \mathrm{~h}$, $12 \mathrm{~h}$, and $24 \mathrm{~h}$ were counted from the photographs.

\section{Apoptosis}

To assess the effect of different treatments on cell apoptosis, treated CPCs were doubly stained with Annexin V-FITC and PI and analyzed by flow cytometry. CPCs $\left(1 \times 10^{5}\right.$ cells) from each group (CPCs cultured in different culture medium for $12 \mathrm{~h}$; negative control (NC) - DMEM medium with $1 \%$ FBS; positive control (PC) - DMEM medium with $10 \%$ FBS; CC-Exos group (CC-Exos)DMEM medium with $1 \% \mathrm{FBS}$ and $30 \mu \mathrm{g} / \mathrm{mL}$ CC-Exos; BMSC-Exos group (BMSC-Exos) - DMEM with 1\% FBS and $30 \mu \mathrm{g} / \mathrm{mL}$ BMSC-Exos) were incubated with FITC-labeled Annexin V and propidium iodide (BD Biosciences, San Jose, CA, USA) for $15 \mathrm{~min}$ at room temperature in the dark. The percentage of apoptotic cells was determined by flow cytometry (BD FACSCalibur, Beckman Coulter).

\section{In vitro angiogenesis assay}

In vitro tubular formation assay was conducted using Matrigel (BD Bioscience, Oxford, UK). The Matrigel was coated onto 48 -well plates at $37{ }^{\circ} \mathrm{C}$ for $30 \mathrm{~min}$ to 
complete gelation according to the manufacturer's instructions. Human umbilical vein endothelial cells (HUVECs) were seeded into pretreated plates, and 1\% FBS, $10 \%$ FBS, or $30 \mu \mathrm{g} / \mathrm{mL}$ of different exosomes were added to each well. After $3 \mathrm{~h}$, cells were photographed using an inverted light microscope. Triplicate samples were tested for each condition, and five random microscopic images were collected for the measurement.

\section{Statistical analysis}

Numerical data are presented as the mean \pm standard deviation (SD) and were analyzed with a one-way ANOVA followed by Tukey's post hoc test. Statistical analysis was performed using GraphPad Prism version 5.0 (GraphPad Software, San Diego, CA, USA). Among the various groups, $P<0.05$ was considered to indicate a significant difference.

\section{Results}

\section{Characterization of BMSC-Exos and CC-Exos}

TEM clearly revealed that the exosomes purified from CCs and BMSCs both showed a cup-shaped or round-shaped form with a diameter of 30-200 nm, which was verified by the Nano-Sight analysis (Fig. 1a). Exosome-associated markers, CD9, CD63, and CD81, were shown by Western blot (Fig. 1b). The above results showed two kinds of exosomes were successfully isolated. In addition, fluorescence microscope images revealed CM Dil-labeled exosomes in the cytoplasm of the CPCs (Fig. 1c), confirming the successful internalization of both kinds of exosomes at $12 \mathrm{~h}$.

\section{CC-Exos promotes ectopic cartilage regeneration}

The effect of CC-Exos on promoting chondrogenesis of CPCs was evaluated in subcutaneous non-chondrogenic sites. At week 4, the CPC constructs supplied with PBS did not lead to noticeable cartilage matrix deposition. The addition of CC-Exos improved the matrix formation, which demonstrated similar morphological characteristics to those in native cartilage, with chondrocytes located within typical chondrocytes lacunae and surrounded by abundant cartilaginous matrix (Fig. 2). In addition, BMSC-Exos, as expected, also improved the cartilage regeneration of the CPC constructs. By 12 weeks, the BMSC-Exos and CC-Exos groups could maintain their cartilage-like appearance, and the histological results revealed significantly more contiguous cartilage matrix deposition than was observed at 4 weeks in each group. The new cartilage tissue exhibited intense staining of both S-F and T-B and showed cartilage-like tissue with typical histological structure and specific matrix deposition. More intense staining was noted in the CC-Exos and BMSC-Exos groups compared with the PBS group. In addition, the expression of typical cartilage markers such as COL II and SOX-9 was significantly enhanced in the BMSC-Exos and CC-Exos groups after 12 weeks (Fig. 3a, b), which indicated superior matrix formation in the implants.

\section{CC-Exos are conducive to maintain the phenotypic stability of engineered cartilage}

We next explored whether the two kinds of exosomes were sufficient to steer the chondrogenesis of engineered tissues toward permanent cartilage-like tissues. Importantly, CPC constructs in the CC-Exos groups stained negatively for COL X (Fig. 3a), indicating tissue hypertrophy. However, implants in the BMSC-Exos group stained intensely for COL X. It is noteworthy that the hypertrophic cartilage-enriched markers of COL X, MMP 13, and IHH were strongly upregulated under long-term use of BMSC-Exos compared with CC-Exos (Fig. 3c). These observations suggested that CC-Exos are conducive to maintain the phenotypic stability of engineered tissue compared with BMSC-Exos.

Leijten reported that hypertrophic differentiation and subsequent calcification are associated with vascular invasion [44]. The angiogenesis in the engineered tissues was then investigated. In gross, the implants in the BMSC-Exos group appeared macroscopically vascularized, whereas CC-Exos implants were predominantly avascular, with some tiny blood vessels visible only in discrete peripheral regions (Fig. 3d). In fact, CD31 staining demonstrated the devoid presence of blood vessels in CC-Exos group. However, the MVD value (microvessels/hotspot) in the BMSC-Exos group was the highest among the three groups $(P<0.05$, Fig. $3 \mathrm{~d})$. These results were further confirmed by the expression of angiogenic hallmarks, such as SDF-1 and VEGF, which were higher in samples from the BMSC-Exos group than those from the CC-Exos group (Fig. 3e).

\section{Exosome uptake and promote CPC proliferation and migration}

To strengthen our in vivo findings, we next analyzed the underlying mechanism in vitro by assessing the effect of exosomes on the migration, proliferation, and matrix synthesis of CPCs.

There was no difference between the two kinds of exosomes internalized by $\mathrm{CPCs}$ according to the mean fluorescence intensity of the same number of cells (Fig. 4a). At a concentration of $30 \mu \mathrm{g} / \mathrm{mL}$, both BMSC-Exos and CC-Exos can stimulate CPC proliferation when compared to $\mathrm{NC}(P<0.05$, Fig. 4c). Furthermore, BMSC-Exos had a much stronger effect on CPC proliferation than CC-Exos $(P<0.05)$, whereas at a concentration of $10 \mu \mathrm{g} / \mathrm{mL}$, there were no marked differences among the CC-Exos, BMSC-Exos, and NC groups $(P>0.05)$. 
a
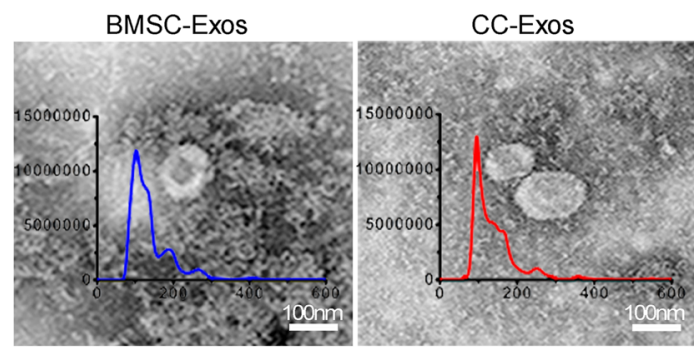

b

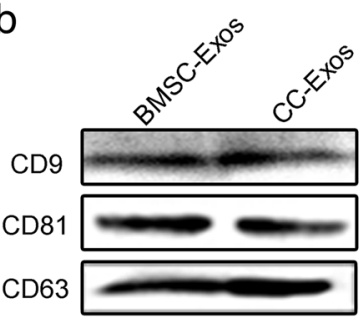

C

DAPI

CM-Dil
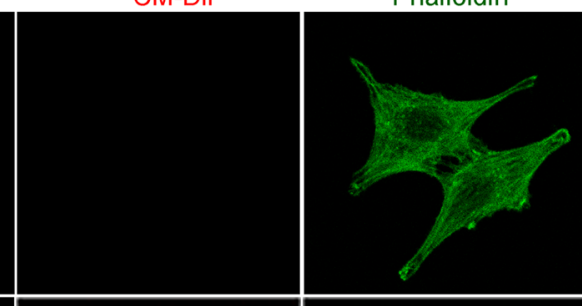

Phalloidin
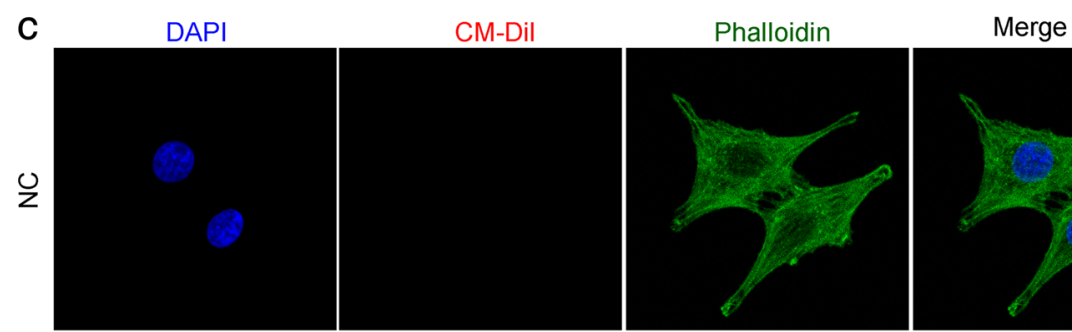
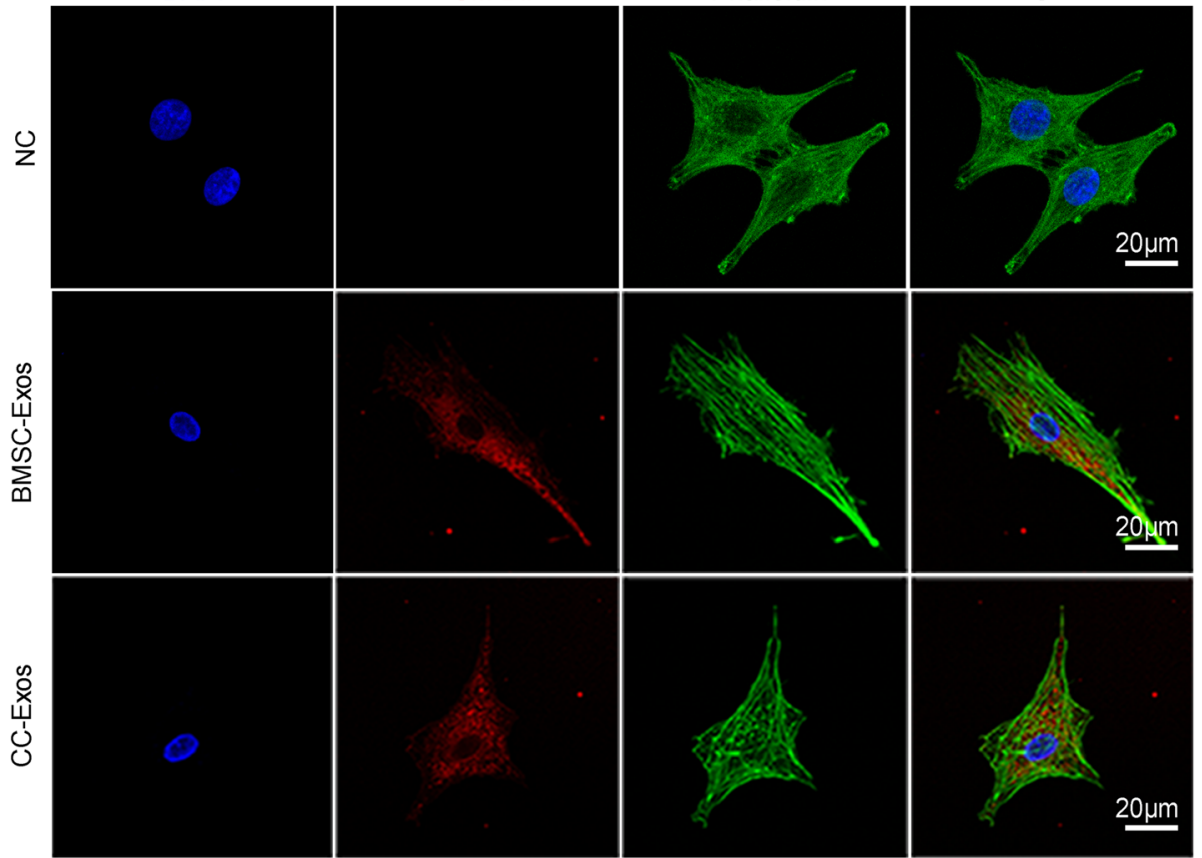

Fig. 1 Characterization of BMSC-Exos and CC-Exos. a Nano-Sight measurement of exosome size and distribution and morphology of exosomes under transmission electron microscopy. Scale bars $=100 \mathrm{~nm}$. b Western blot analysis of exosome-specific markers CD9, CD63, and CD81. c Representative immunofluorescence photomicrograph of CM-Dil (red)-labeled exosomes absorbed by CPCs at $12 \mathrm{~h}$, the F-actin of which were stained by phalloidin (green), and the nuclei were stained by DAPI (blue). Scale bars $=20 \mu \mathrm{m}$

To further assess the different exosome-induced CPC proliferation, nuclear Ki67 staining was carried out as a surrogate for CPC proliferation (Fig. 4b). After incubating CPCs for $12 \mathrm{~h}$ on the substrates, $10 \mu \mathrm{g} / \mathrm{mL}$ of both BMSC-Exos and CC-Exos had a higher Ki67 expression than the negative control, which was more evident at $30 \mu \mathrm{g} / \mathrm{mL}$. These results suggest that both CC-Exos and BMSC-Exos increase the mitogenic effect of the CPCs.

An in vitro transwell assay was performed to investigate the exosome-stimulating effects of migration on CPCs. CC-Exos did not stimulate migration of CPCs after $12 \mathrm{~h}(P$ $<0.05$, Fig. 5a, b). In contrast, CPC migration increased significantly in the presence of BMSC-Exos after 6 and $12 \mathrm{~h}$, when compared with the NC or CC-Exos groups $(P<0.05)$, and the number of migrated cells approached the number identified in the PC group $(P>0.05)$. We also performed a cell apoptosis analysis at $12 \mathrm{~h}$, which revealed no significant difference between the four groups (Fig. 5c). Based on these findings, we believe the different migration cell number was attributed to the different chemotaxis effect of the exosomes. Scratch wound assays further proved that BMSC-Exos were more effective than CC-Exos in increasing the motility of CPCs at $24 \mathrm{~h}(P<0.05$, Fig. $5 \mathrm{~d}$, e). The above results confirmed that the extracted BMSC-Exos and CC-Exos could promote the proliferation of CPCs, with BMSC-Exos exerting a stronger effect, while only BMSC-Exos can increase the migration of CPCs.

\section{CC-Exos promote the differentiation of CPCs}

COL II and SOX-9 expression were then evaluated as indicators of chondrogenic differentiation. Importantly, when exposed to two kinds of exosomes, genes associated with chondrogenic differentiation and matrix 


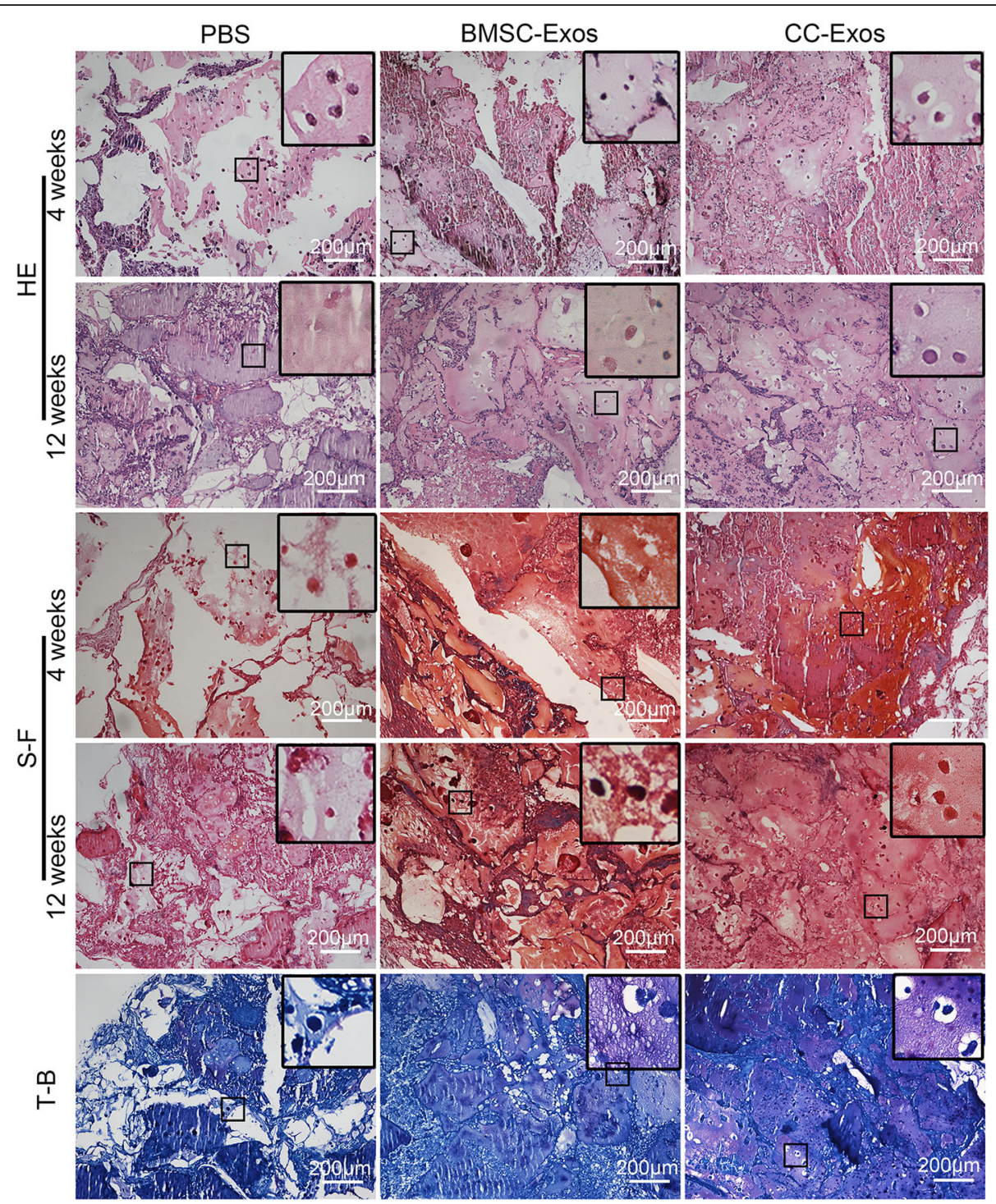

Fig. 2 Histological analysis. H\&E staining, safranin O/fast green (S-F) staining, and toluidine blue (T-B) staining in 4 weeks or 12 weeks showed enhanced ectopic cartilage formation in the BMSC-Exos and CC-Exos groups, compared to the PBS group. The enlarged images detailed the chondrocytes surrounded by a cartilaginous matrix, where more chondrocytes were found in the CC-Exos group, followed by BMSC-ExOs and PBS groups. At week 4, the CPC constructs supplied with PBS did not lead to obvious cartilage matrix deposition. The addition of both BMSC-Exos and CC-Exos improved the matrix formation, compared with the PBS group. By 12 weeks, the BMSC-Exos and CC-Exos groups could maintain their cartilage-like appearance, and the histological results revealed significantly more contiguous cartilage matrix deposition than was observed at 4 weeks in each group. Black rectangles: typical chondrocytes, which are in high magnification. Scale bars $=200 \mu \mathrm{m}$

synthesis, such as COL II and SOX-9, were significantly increased at the mRNA levels, with the CC-Exos exerting a stronger effect $(P<0.05$, Fig. $4 \mathrm{~d})$. Since TGF- $\beta$ signaling pathway is one of the main pathways to determine the chondrogenesis $[45,46]$, we also performed a preliminary study on the expression change of TGF- $\beta$ signaling pathway after BMSC-Exos and CC-Exos treatment. CC-Exos stimulated the protein expression of TGF- $\beta$ and SMAD2/3 (Fig. 4e). The downstream expression of COL II and SOX 9 were also increased. Thus, CC-Exos internalized by CPCs exerted chondrogenic effects possibly through the TGF- $\beta /$ SMAD signaling pathway.

Furthermore, the factors involved in vascularization, such as VEGF and SDF-1, were significantly increased at the mRNA and protein levels in the BMSC-Exos group compared to the $\mathrm{NC}$ and CC-Exos group $(P<0.05$, Fig. 4d, f). Interestingly, the mRNA levels of VEGF and SDF-1 in CPCs cultured with CC-Exos were lower than those in CPCs cultures with $\mathrm{NC}(P<0.05)$, but the protein levels of the two groups had no discernible difference. 


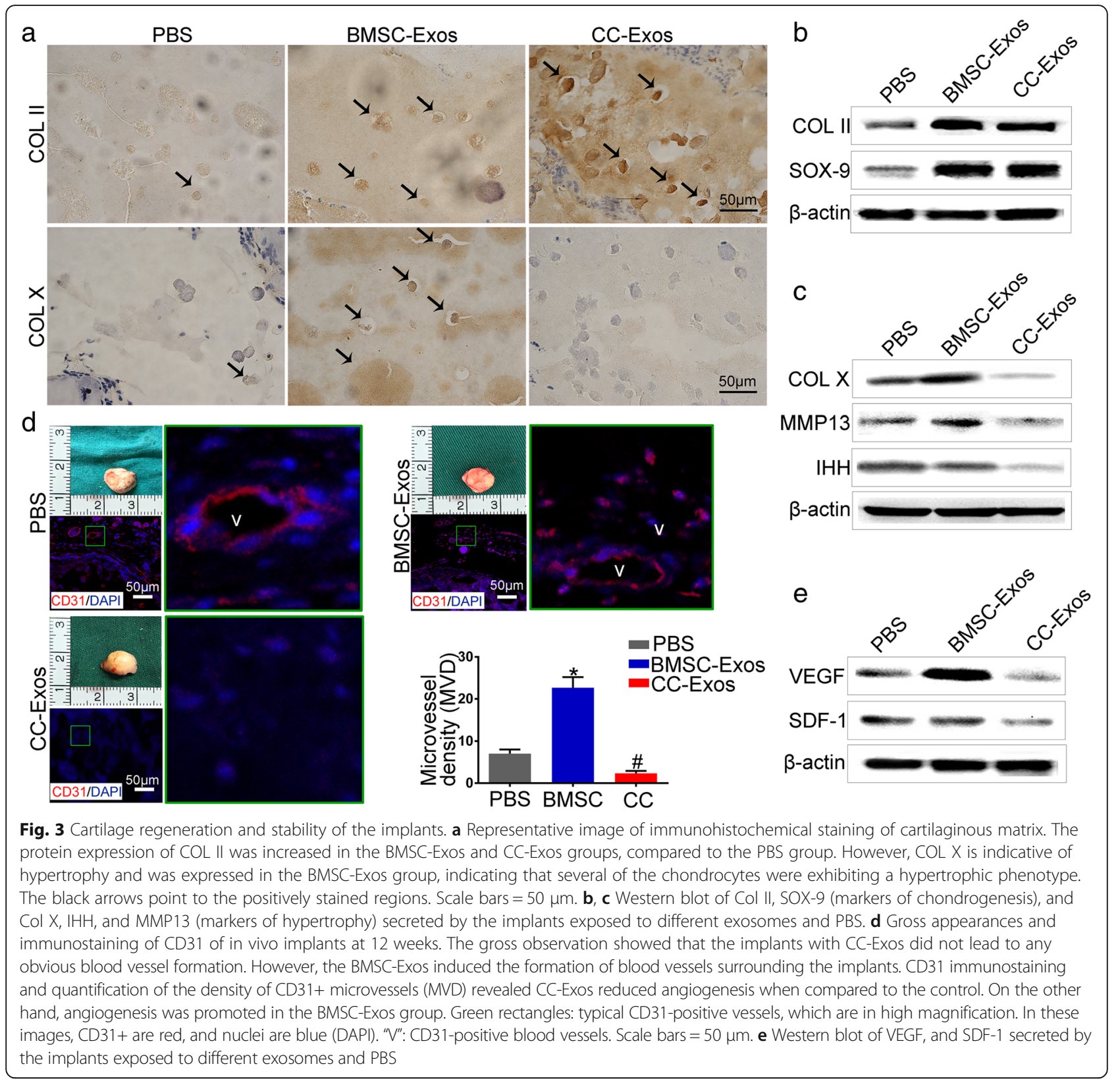

\section{BMSC-Exos and not CC-Exos promote HUVEC migration} and angiogenesis

An in vitro transwell assay was performed to investigate the exosome-stimulating effects on HUVECs. HUVEC migration increased significantly in the presence of BMSC-Exos, compared to the NC and CC-Exos group $(P<0.05$, Fig. 6a, b), and the number of migrated cells approached the number identified in the $\mathrm{PC}$ group $(P>$ 0.05). However, CC-Exos had no significant effect on HUVEC migration, even compared with the NC group $(P>0.05)$.

Furthermore, the classical Matrigel assay demonstrated that BMSC-Exos exhibited the capacity to promote the formation of capillary-like structures compared with the NC or CC-Exos group $(P<0.05$, Fig. $6 c-e)$. Contrary to the angiogenic role of BMSC-Exos, the CC-Exos produced shorter networks with the least number of mesh, values that were not significantly different than the NC group $(P>0.05)$.

In short, these findings indicate that although BMSC-Exos could promote CPCs proliferation and migration, it was also prone to angiogenesis and hypertrophic differentiation. On the other hand, the CC-Exos displayed a robust chondral matrix formation with minor angiogenesis, which circumvented the inherent drawbacks of BMSC-Exos. The results further 


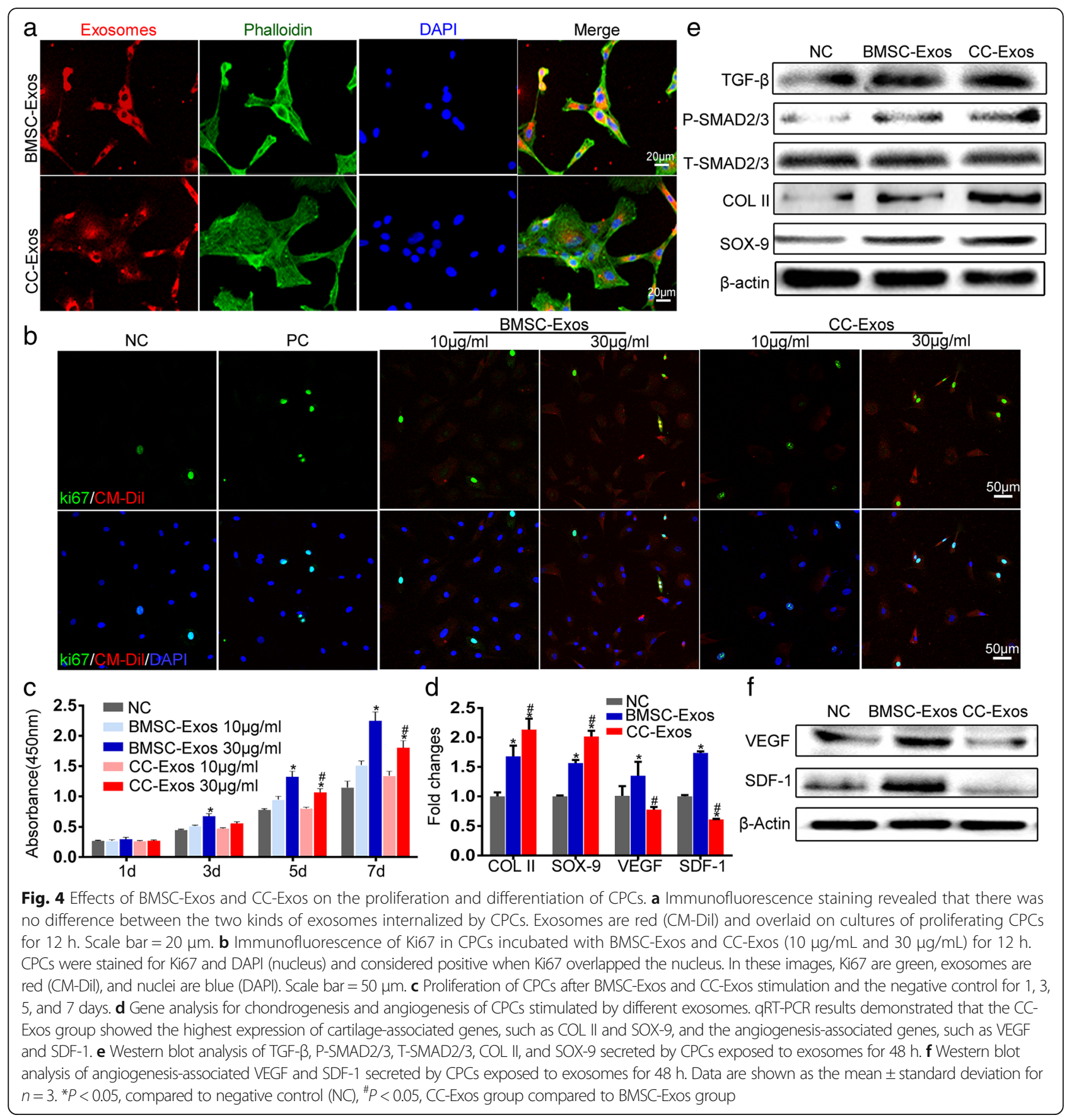

corroborated its preferable cartilage regeneration and stability of CC-Exos in vivo.

\section{Discussion}

Cartilage defect treatment represents a major clinical problem worldwide because of the increasing incidences of trauma, disease, or aging $[47,48]$. In clinics, autogenous cartilage is typically required to implant to the defect sites in order to restore normal appearance and function [49-51], which was fraught with a couple of shortcomings [5, 52, 53]. Tissue-engineered cartilage grafts have emerged as a promising alternative to overcome these problems and satisfy the ever-increasing clinical need [54-56]. Currently, exosomes have been identified as the principal agent in mediating the therapeutic efficacy of the cell-based regenerative medicine approach $[20,21,57,58]$, and BMSC-Exos have been reported for promoting in situ cartilage defect repair [21, 26]. However, the therapeutic outcome for subcutaneous cartilage defect repair is still limited 
a

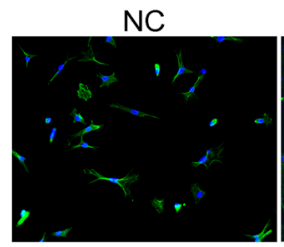

$6 \mathrm{~h}$

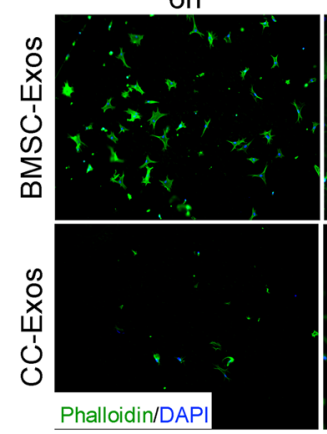

C

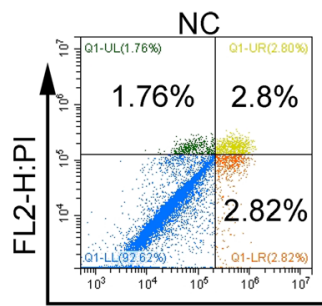

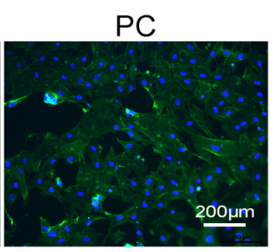

$12 \mathrm{~h}$
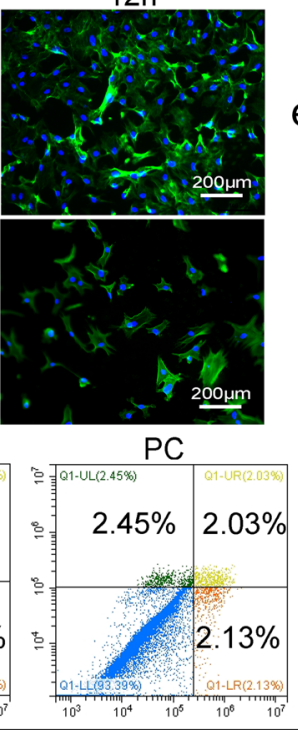
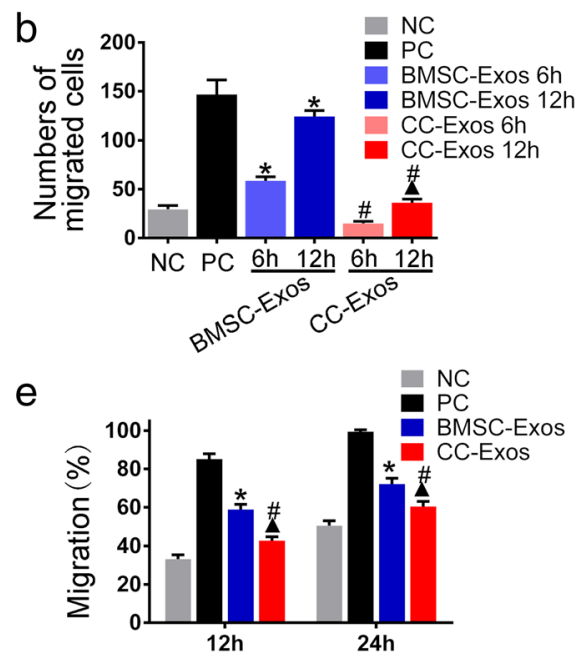

BMSC-Exos

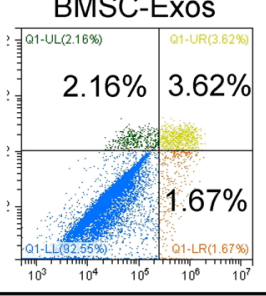

CC-Exos

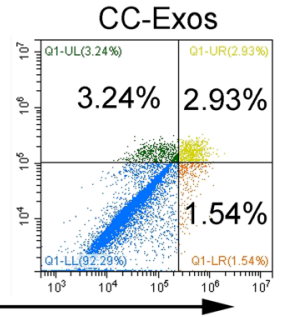

FL1-H:Annexin V-FITC

\section{d}

NC PC BMSC-Exos
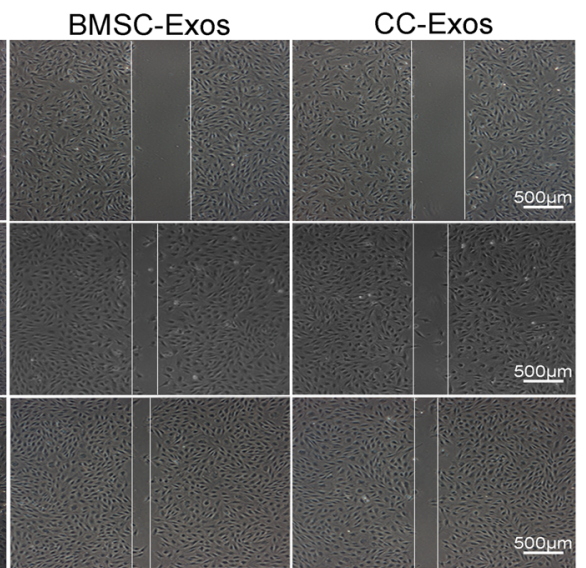

Fig. 5 The effects of exosomes on CPCs migration. $\mathbf{a}$, b Migration of CPCs exposed to different conditioned medium. Images of NC (negative control, DMEM medium with 1\% FBS), PC (positive control, DMEM medium with 10\% FBS), and CC-Exos and BMSC-Exos group (DMEM medium with $1 \% \mathrm{FBS}$ and $30 \mathrm{\mu g} / \mathrm{mL}$ exosomes) show result of cell migration at $12 \mathrm{~h}$ and quantitative analysis of migrated cells. Exosomes are red, phalloidin is green, and nuclei are blue. Scale bar $=200 \mu \mathrm{m}$. c The apoptosis level of CPCs incubated with BMSC-Exos and CC-Exos for 12 h. Apoptotic cells were stained by PI/Annexin- $V$ followed by flow cytometry. $\mathbf{d}$, e Light microscopy images of the scratch wound assays and quantitative analysis of migration rates at $12 \mathrm{~h}$ and $24 \mathrm{~h}$. Scale bar $=200 \mu \mathrm{m}$. ${ }^{*} P<0.05$, compared to negative control (NC), ${ }^{\boldsymbol{\Delta}} P<0.05$, compared to positive control (PC), ${ }^{\#} P<0.05, C C$ Exos group compared to BMSC-Exos groups

because of the lack of a suitable pro-chondrogenic environment [17, 40,59]. Meanwhile, previous studies have also shown that chondrocytes could steer the chondrogenesis of stem cells in vitro and in vivo through paracrine effects $[1,7,60]$. In the present study, using a CPC-based cartilage tissue engineering approach, the potential of CC-Exos in promoting ectopia chondrogenesis and stabilizing cartilage regeneration in a subcutaneous environment was further investigated.

The current study demonstrated that CPC constructs supplied with CC-Exos could form homogeneous cartilage-like tissue with minimal hypertrophy in a subcutaneous environment, with no help from any 


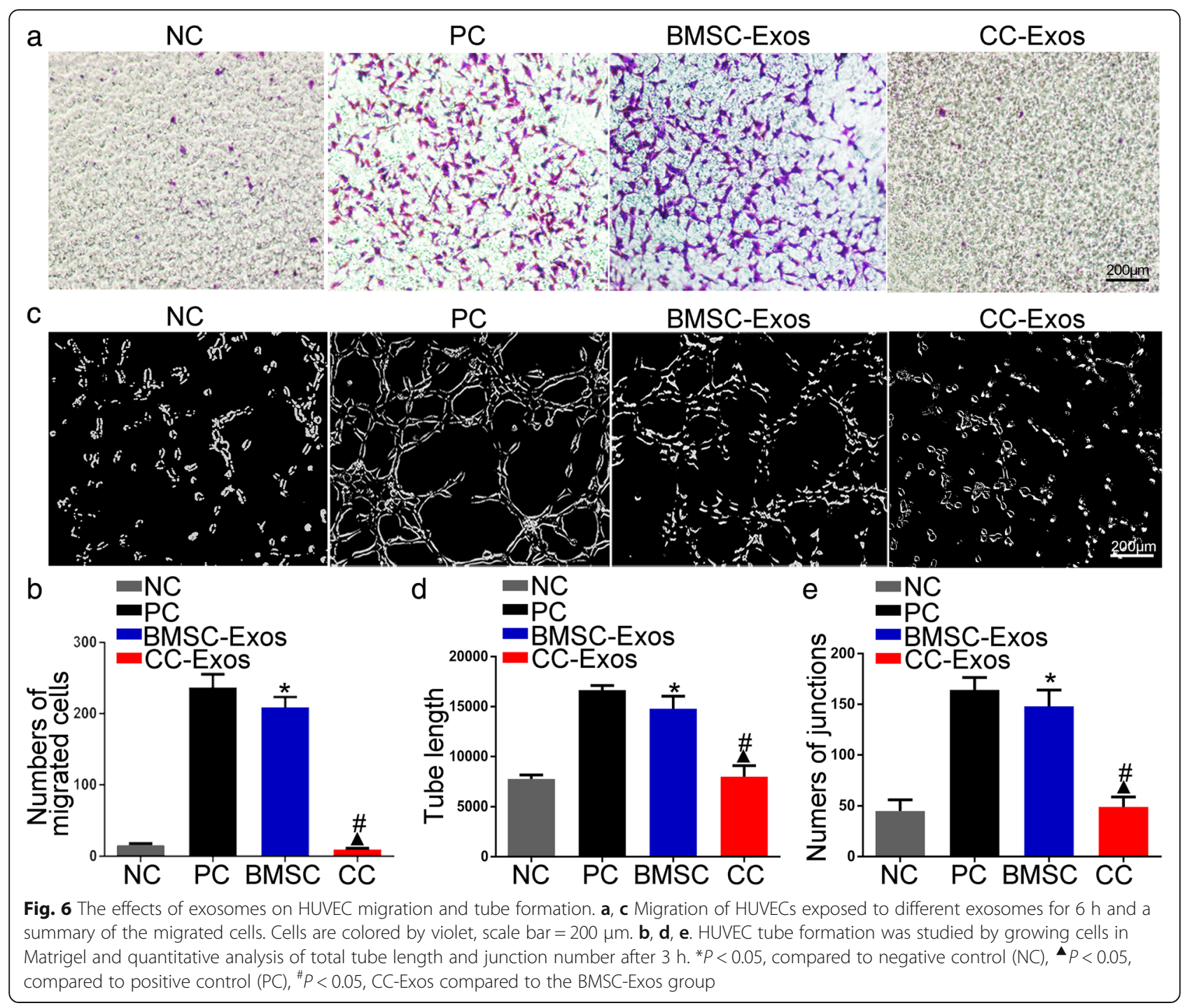

chondrogenic factors. Furthermore, a series of in vitro experiments further confirmed that CC-Exos significantly promoted chondrogenesis-related factors at the mRNA and protein levels in CPCs, such as SOX-9 and COL II. Importantly, angiogenesis was inhibited by CC-Exos, which is known to be detrimental to cartilage regeneration leading to hypertrophic differentiation and subsequent calcification [44]. The observed contributions of CC-Exos to cartilage regeneration in vivo corroborate the in vitro findings and further support that CC-Exos alone could provide a preferable chondrogenic environment and help maintain the stability of cartilage tissue. Compared with BMSC-Exos where samples show more hypertrophic cartilage, the cartilage regeneration results achieved with the use of CC-Exos are significantly more favorable. Hence, the null hypothesis that there is no difference between CC-Exos and BMSC-Exos in cartilage regeneration results must be rejected.
To date, tissue engineering has offered promising solutions for clinical issues involving congenital and acquired cartilage defects [7, 61, 62]. However, the cartilage formation in subcutaneous environments is limited due to the lack of a proper chondrogenic niche [11, 12, 59]. Imitating the chondrogenic niche is a well-accepted approach to promote the ectopic chondrogenesis of progenitor cells $[16,18]$. Exosomes have been studied extensively for their potential in participating in the maintenance of normal physiology via delivering various types of bioactive microRNAs, nucleic acids, proteins, and unique gene products [22,63]. Recent studies have shown that chondrocytes and chondrocyte-related factors play key regulatory roles in the maintenance of the cartilage microenvironment and the ultimate cartilage phenotype of implanted stem cells [13-15]. In the present study, we further demonstrate that CC-Exos modulates $\mathrm{CPC}$ migration, proliferation, and cartilage 
matrix synthesis. Expression of SOX-9 and COL II by CPCs is upregulated in the presence of CC-Exos, which promotes chondrogenesis. This may be attributed to the TGF- $\beta$ /SMAD signaling pathway, which is reported to play an essential role in chondrocyte differentiation and matrix maturation [41, 45, 64]. More investigations are needed to acquire the whole picture of the pathway involved in CC-Exos-induced chondrogenesis.

Additionally, reproducibly generating stable cartilage remains an unsolved challenge. Avoiding vessel ingrowth and hypertrophy is a critical factor in building stable cartilage $[65,66]$. In the present study, compared to the positive control groups (BMSC-Exos), CC-Exos could maintain a stabilized phenotype of constructed cartilage at least within the investigated time frame, as evidenced by the presence of significantly less COL X-positive staining and minimal protein expression of $\mathrm{COL} \mathrm{X}, \mathrm{IHH}$, and MMP 13 secreted by hypertrophic chondrocytes at 12 weeks. In addition, less CD31-positive microvessels are observed in the neo-cartilage of the CC-Exos group. However, after the addition of BMSC-Exos, expression of SDF-1 and VEGF is upregulated, which promotes cell homing and angiogenesis. This is beneficial for cartilage engineering during the early stage of implantation [40], which may account for the better neo-cartilage formation in the BMSC-Exos, as reported [21, 26]. However, it also has a disadvantage as evidenced by promoting associated ectopic cartilage hypertrophy. Recent studies have also shown that vascular invasion is one of the major mechanisms involved in hypertrophic cartilage differentiation [5, 44]. In vitro results also revealed that HUVEC migration and tube formation are reduced by CC-Exos when compared with BMSC-Exos. These results collaborate with data collected from an in vivo experiment, which shows CC-Exos have the ability to decrease angiogenesis in subcutaneous cartilage repair. Because CC-Exos can promote CPC migration, proliferation, and matrix synthesis in vitro, a more favorable prognosis is anticipated for long-term cartilage regeneration.

A novel method of imitating the chondrogenic niche is explored in the present work via the use of CC-Exos. After local injection of CC-Exos, the CPCs are rapidly directed to form neo-cartilage, when stimulated by chondroinductive mediators. Importantly, the engineered cartilage here can maintain the stabilized phenotype in non-chondrogenic niches, which is probably related to antiangiogenic factors secreted by CC-Exos that prevent neovascularization and hypertrophy. Because strategies that provide the conventional cartilage environment often require cell-based therapy [54, 55], the use of CC-Exos is advantageous from the perspectives of off-the-shelf and cell-free regenerative medicine approach for cartilage repair, and the ease of minimally invasive injection of CC-Exos concentrate.
Despite these encouraging results, the exact component(s) is yet to be elucidated. It is plausible that a myriad of components is present in the CC-Exos that can orchestrate cartilage regeneration including chondrogenesis and stability. However, the detailed mechanism of CC-Exos treatment to CPCs that caused the difference from that of BMSC-Exos is still unclear, and further investigation of RNA-seq is needed to dissect the components present in CC-Exos and to investigate their underlying mechanisms in cartilage repair. In addition, chondrogenesis is a complex process which is related to various signaling pathways, such as wnt, TGF- $\beta$, and hedgehog pathway. Here, we preliminarily demonstrated the induction of TGF- $\beta$ and downstream SMAD2/3 expression after CC-Exos treatment. Further investigation is needed to acquire the entire picture of the pathway.

\section{Conclusions}

In summary, this study demonstrated that a novel exosome from chondrocytes could imitate the chondrogenic niche in a subcutaneous environment, which could facilitate chondrogenesis and maintenance of cartilage stability. This may contribute to its preferable chondroinductive niches coupled with its antiangiogenic properties. Thus, CC-Exos may represent a promising biologic-based therapeutic approach for the treatment of ectopic cartilage defects.

\section{Abbreviations \\ BMSC: Bone mesenchymal stem cells; BMSC-Exos: Exosomes secreted by bone mesenchymal stem cells; CC-Exos: Exosomes secreted by chondrocytes: COL II: Collagen type II; COL X: Collagen type X; CPCs: Cartilage progenitor/ stem cells; DMEM: Dulbecco's modified Eagle's medium; FBS: Fetal bovine serum; H\&E: Hematoxylin and eosin; HUVECs: Human umbilical vein endothelial cells; $\mid \mathrm{HH}$ : Indian hedgehog; MMP 13: Matrix metalloproteinase 13; NC: Negative control; PBS: Phosphate-buffered saline; PC: Positive control; RT-PCR: Real-time quantitative polymerase chain reaction; SDF-1: Stem cell- derived factor 1; S-F: Safranin-O/fast green; T-B: Toluidine blue; \\ TEM: Transmission electron microscopy; TGF- $\beta$ : Transforming growth factor- $\beta$; VEGF: Vascular endothelial growth factor}

\section{Acknowledgements}

Not applicable.

\section{Funding}

This research was supported by the National Natural Science Foundation of China $(81471878,81272128,30872697)$.

\section{Availability of data and materials}

All data generated or analyzed during this study are included in this published article. For additional information, please contact the author.

\section{Authors' contributions}

YHC, ZWZ, and KL conceived the idea, designed the experiments, provided their funds for the study, and revised the manuscript. YHC and ZWZ designed the study and performed the research, data analysis, and manuscript writing. KX and XDZ contributed to the analyses and interpretation of data. All authors read and approved the final manuscript for publication.

Ethics approval and consent to participate

All procedures of the animal experiments were approved by the Animal Research Committee of Shanghai Jiao Tong University Affiliated Ninth People's Hospital (No. HKDL 2017-132). 


\section{Consent for publication}

Not applicable.

\section{Competing interests}

The authors declare that they have no competing interests.

\section{Publisher's Note}

Springer Nature remains neutral with regard to jurisdictional claims in published maps and institutional affiliations.

\section{Author details}

'Department of Plastic and Reconstructive Surgery, Shanghai Ninth People's Hospital, College of Stomatology, Shanghai Jiao Tong University School of Medicine, 639 ZhiZaoJu Road, Shanghai 200011, People's Republic of China. ${ }^{2}$ Department of Oral and Maxillofacial-Head and Neck Oncology, Shanghai Ninth People's Hospital, College of Stomatology, Shanghai Jiao Tong University School of Medicine, 639 ZhiZaoJu Road, Shanghai 200011 , People's Republic of China. ${ }^{3}$ National Clinical Research Center for Oral Diseases, 639 ZhiZaoJu Road, Shanghai 200011, People's Republic of China. ${ }^{4}$ Shanghai Key Laboratory of Stomatology \& Shanghai Research Institute of Stomatology, 639 ZhiZaoJu Road, Shanghai 200011, People's Republic of China.

Received: 9 May 2018 Revised: 30 July 2018 Accepted: 16 October 2018 Published online: 21 November 2018

\section{References}

1. Liu X, Sun H, Yan D, Zhang L, Lv X, Liu T, et al. In vivo ectopic chondrogenesis of BMSCs directed by mature chondrocytes. Biomaterials. 2010;31:9406-14.

2. He A, Xia H, Xiao K, Wang T, Liu Y, Xue J, et al. Cell yield, chondrogenic potential, and regenerated cartilage type of chondrocytes derived from ear, nasoseptal, and costal cartilage. J Tissue Eng Regen Med. 2018;12:1123-32.

3. Kawanabe $Y$, Nagata S. A new method of costal cartilage harvest for total auricular reconstruction: part I. Avoidance and prevention of intraoperative and postoperative complications and problems. Plast Reconstr Surg. 2006; 117:2011-8.

4. Padilla S, Sanchez M, Orive G, Anitua E. Human-based biological and biomimetic autologous therapies for musculoskeletal tissue regeneration. Trends Biotechnol. 2017:35:192-202.

5. Huey DJ, Hu JC, Athanasiou KA. Unlike bone, cartilage regeneration remains elusive. Science. 2012;338:917-21.

6. Johnstone B, Alini M, Cucchiarini M, Dodge GR, Eglin D, Guilak F, et al. Tissue engineering for articular cartilage repair - the state of the art. Eur Cells Mater. 2013;25:248-67.

7. Leijten JC, Georgi N, Wu L, van Blitterswijk CA, Karperien M. Cell sources for articular cartilage repair strategies: shifting from monocultures to cocultures. Tissue Eng Part B Rev. 2013;19:31-40.

8. Demoor M, Ollitrault D, Gomez-Leduc T, Bouyoucef M, Hervieu M, Fabre H, et al. Cartilage tissue engineering: molecular control of chondrocyte differentiation for proper cartilage matrix reconstruction. Biochim Biophys Acta. 2014:1840:2414-40

9. Shen W, Chen J, Zhu T, Chen L, Zhang W, Fang Z, et al. Intra-articular injection of human meniscus stem/progenitor cells promotes meniscus regeneration and ameliorates osteoarthritis through stromal cell-derived factor-1/CXCR4-mediated homing. Stem Cells Transl Med. 2014;3:387-94.

10. Mizuno M, Kobayashi S, Takebe T, Kan H, Yabuki Y, Matsuzaki T, et al. Brief report: reconstruction of joint hyaline cartilage by autologous progenitor cells derived from ear elastic cartilage. Stem Cells. 2014;32:816-21.

11. Pelttari K, Winter A, Steck E, Goetzke K, Hennig T, Ochs BG, Aigner T, Richter $W$. Premature induction of hypertrophy during in vitro chondrogenesis of human mesenchymal stem cells correlates with calcification and vascular invasion after ectopic transplantation in SCID mice. Arthritis Rheum. 2006;54: 3254-66.

12. Liu K, Zhou GD, Liu W, Zhang WJ, Cui L, Liu X, et al. The dependence of in vivo stable ectopic chondrogenesis by human mesenchymal stem cells on chondrogenic differentiation in vitro. Biomaterials. 2008:29:2183-92.

13. Becerra J, Santos-Ruiz L, Andrades JA, Mari-Beffa M. The stem cell niche should be a key issue for cell therapy in regenerative medicine. Stem Cell Rev. 2011;7:248-55.
14. Lane SW, Williams DA, Watt FM. Modulating the stem cell niche for tissue regeneration. Nat Biotechnol. 2014;32(8):795-803.

15. Jayasuriya $C T, C$ Chen $Y, L i u$ W, Chen $Q$. The influence of tissue microenvironment on stem cell-based cartilage repair. Ann N Y Acad Sci. 2016;1383:21-33.

16. Leyh $M$, Seitz $A$, Dürselen $L$, Springorum $H-R$, Angele $P$, Ignatius $A$, et al. Osteoarthritic cartilage explants affect extracellular matrix production and composition in cocultured bone marrow-derived mesenchymal stem cells and articular chondrocytes. Stem Cell Res Ther. 2014:5:77.

17. Li D, Zhu L, Liu Y, Yin Z, Liu Y, Liu F, et al. Stable subcutaneous cartilage regeneration of bone marrow stromal cells directed by chondrocyte sheet. Acta Biomater. 2017:54:321-32.

18. Zhao X, Hwang NS, Bichara DA, Saris DB, Malda J, Vacanti JP, et al. Chondrogenesis by bone marrow-derived mesenchymal stem cells grown in chondrocyte-conditioned medium for auricular reconstruction. J Tissue Eng Regen Med. 2017;11:2763-73.

19. Ahmed N, Dreier R, Gopferich A, Grifka J, Grassel S. Soluble signalling factors derived from differentiated cartilage tissue affect chondrogenic differentiation of rat adult marrow stromal cells. Cellular physiology and biochemistry: international journal of experimental cellular physiology, biochemistry, and pharmacology. 2007;20:665-678.

20. Lai RC, Yeo RW, Lim SK. Mesenchymal stem cell exosomes. Semin Cell Dev Biol. 2015;40:82-8

21. Zhang S, Chuah SJ, Lai RC, Hui JHP, Lim SK, Toh WS. MSC exosomes mediate cartilage repair by enhancing proliferation, attenuating apoptosis and modulating immune reactivity. Biomaterials. 2018;156:16-27.

22. Katare R, Stroemer P, Hicks C, Stevanato L, Patel S, Corteling R, et al. Clinical-grade human neural stem cells promote reparative neovascularization in mouse models of hindlimb ischemia. Arterioscler Thromb Vasc Biol. 2014;34:408-18.

23. Jo W, Kim J, Yoon J, Jeong D, Cho S, Jeong H, et al. Large-scale generation of cell-derived nanovesicles. Nanoscale. 2014;6:12056-64.

24. Lee C, Carney RP, Hazari S, Smith ZJ, Knudson A, Robertson CS, et al. 3D plasmonic nanobowl platform for the study of exosomes in solution. Nanoscale. 2015;7:9290-7.

25. Colombo M, Raposo G, Thery C. Biogenesis, secretion, and intercellular interactions of exosomes and other extracellular vesicles. Annu Rev Cell Dev Biol. 2014:30:255-89.

26. Toh WS, Lai RC, Hui JHP, Lim SK. MSC exosome as a cell-free MSC therapy for cartilage regeneration: implications for osteoarthritis treatment. Semin Cell Dev Biol. 2017;67:56-64.

27. Melle ML d V-V, Narcisi R, Kops N, BSc WJLMK, et al. Chondrogenesis of mesenchymal stem cells in an osteochondral environment is mediated by the subchondral bone. Tissue Eng Part A. 2014;20:23-33.

28. Tao SC, Yuan T, Zhang YL, Yin WJ, Guo SC, Zhang CQ. Exosomes derived from miR-140-5p-overexpressing human synovial mesenchymal stem cells enhance cartilage tissue regeneration and prevent osteoarthritis of the knee in a rat model. Theranostics. 2017;7:180-95.

29. Cosenza S, Ruiz M, Toupet K, Jorgensen C, Noel D. Mesenchymal stem cells derived exosomes and microparticles protect cartilage and bone from degradation in osteoarthritis. Sci Rep. 2017;7:16214.

30. Zhang S, Chu WC, Lai RC, Lim SK, Hui JH, Toh WS. Exosomes derived from human embryonic mesenchymal stem cells promote osteochondral regeneration. Osteoarthr Cartil. 2016;24:2135-40.

31. Xue K, Xia W, Zhang X, Qi L, Zhou J, Xu P, Liu K. Isolation and identification of stem cells in different subtype of cartilage tissue. Expert Opin Biol Ther. 2015;15:623-32.

32. Dowthwaite GP, Bishop JC, Redman SN, Khan IM, Rooney P, Evans DJ, et al. The surface of articular cartilage contains a progenitor cell population. J Cell Sci. 2004:117:889-97.

33. Xue K, Qi L, Zhou G, Liu K. A two-step method of constructing mature cartilage using bone marrow-derived mesenchymal stem cells. Cells Tissues Organs. 2013;197:484-95.

34. Zhu Y, Wang Y, Zhao B, Niu X, Hu B, Li Q, et al. Comparison of exosomes secreted by induced pluripotent stem cell-derived mesenchymal stem cells and synovial membrane-derived mesenchymal stem cells for the treatment of osteoarthritis. Stem Cell Res Ther. 2017;8:64.

35. Liu X, Wang S, Wu S, Hao Q, Li Y, Guo Z, Wang W. Exosomes secreted by adipose-derived mesenchymal stem cells regulate type I collagen metabolism in fibroblasts from women with stress urinary incontinence. Stem Cell Res Ther. 2018;9:159. 
36. Wang X, Shen H, Zhangyuan G, Huang R, Zhang W, He Q, et al. 14-3-3zeta delivered by hepatocellular carcinoma-derived exosomes impaired anti-tumor function of tumor-infiltrating T lymphocytes. Cell Death Dis. 2018;9:159.

37. Arlov O, Steinwachs M, Skjak-Braek G, Zenobi-Wong M. Biomimetic sulphated alginate hydrogels suppress IL-1 beta-induced inflammatory responses in human chondrocytes. Eur Cell Mater. 2017;33:76-89.

38. Chen CY, Rao SS, Ren L, Hu XK, Tan YJ, Hu Y, et al. Exosomal DMBT1 from human urine-derived stem cells facilitates diabetic wound repair by promoting angiogenesis. Theranostics. 2018;8:1607-23.

39. Zhang B, Wang M, Gong A, Zhang $X$, Wu X, Zhu Y, et al. HucMSC-exosome mediated-Wnt4 signaling is required for cutaneous wound healing. Stem Cells. 2015;33:2158-68.

40. Li Z, Ba R, Wang Z, Wei J, Zhao Y, Wu W. Angiogenic potential of human bone marrow-derived mesenchymal stem cells in chondrocyte brickenriched constructs promoted stable regeneration of craniofacial cartilage. Stem Cells Transl Med. 2017;6:601-12.

41. Wang W, Qu M, Xu L, Wu X, Gao Z, Gu T, et al. Sorafenib exerts an antikeloid activity by antagonizing TGF-beta/Smad and MAPK/ERK signaling pathways. J Mol Med (Berl). 2016;94:1181-94.

42. Sun $J$, Jiao K, Niu LN, Jiao Y, Song $Q$, Shen $L$, et al. Intrafibrillar silicified collagen scaffold modulates monocyte to promote cell homing, angiogenesis and bone regeneration. Biomaterials. 2017;113:203-16.

43. Pan Y, Wu Q, Qin L, Cai J, Du B. Gold nanoparticles inhibit VEGF165-induced migration and tube formation of endothelial cells via the Akt pathway. Biomed Res Int. 2014;16:418624

44. Leijten J, Georgi N, Moreira Teixeira L, van Blitterswijk CA, Post JN, Karperien M. Metabolic programming of mesenchymal stromal cells by oxygen tension directs chondrogenic cell fate. Proc Natl Acad Sci U S A. 2014;111: 13954-9.

45. Chiang CS, Chen JY, Chiang MY, Hou KT, Li WM, Chang SJ, Chen SY. Using the interplay of magnetic guidance and controlled TGF-beta release from protein-based nanocapsules to stimulate chondrogenesis. Int J Nanomedicine. 2018;13:3177-88.

46. Chen MJ, Whiteley JP, Please CP, Schwab A, Ehlicke F, Waters SL, et al. Inducing chondrogenesis in MSC/chondrocyte co-cultures using exogenous TGF-beta: a mathematical model. J Theor Biol. 2018;439:1-13.

47. Yang J, Zhang YS, Yue K, Khademhosseini A. Cell-laden hydrogels for osteochondral and cartilage tissue engineering. Acta Biomater. 2017;57: $1-25$

48. Du Y, Liu H, Yang Q, Wang S, Wang J, Ma J, et al. Selective laser sintering scaffold with hierarchical architecture and gradient composition for osteochondral repair in rabbits. Biomaterials. 2017;137:37-48.

49. Kawanabe $Y$, Nagata S. A new method of costal cartilage harvest for total auricular reconstruction: part II. Evaluation and analysis of the regenerated costal cartilage. Plast Reconstr Surg. 2007;119:308-15.

50. Ou LF, Yan RS, Tang YW. Firm elevation of the auricle in reconstruction of microtia with a retroauricular fascial flap wrapping an autogenous cartilage wedge. Br J Plast Surg. 2001;54:573-80.

51. Dakak M, Gurkok S, Gozubuyuk A, Genc O. Surgical repair of congenital sternal cleft in an adult to prevent traumatic pericardial rupture. Thorac Cardiovasc Surg. 2006:54:551-3.

52. Del Frari B, Schwabegger AH. Diced autologous rib cartilage for primary treatment or refinement of minor chest wall deformities. Plast Reconstr Surg. 2011;128:154-62.

53. Haisch A, Klaring S, Groger A, Gebert C, Sittinger M. A tissue-engineering model for the manufacture of auricular-shaped cartilage implants. Eur Arch Otorhinolaryngol. 2002;259:316-21.

54. Huang BJ, Hu JC, Athanasiou KA. Cell-based tissue engineering strategies used in the clinical repair of articular cartilage. Biomaterials. 2016;98:1-22.

55. Brunelle AR, Horner CB, Low K, ICo G, Nam J. Electrospun thermosensitive hydrogel scaffold for enhanced chondrogenesis of human mesenchymal stem cells. Acta Biomater. 2018;6:166-76.

56. Patel JM, Ghodbane SA, Brzezinski A, Gatt CJ, Dunn MG. Tissue-engineered total meniscus replacement with a fiber-reinforced scaffold in a 2-year ovine model. Am J Sports Med. 2018;46:1844-56.

57. Poe AJ, Knowlton AA. Exosomes as agents of change in the cardiovascular system. J Mol Cell Cardiol. 2017;111:40-50.

58. Ribeiro-Rodrigues TM, Laundos TL, Pereira-Carvalho R, Batista-Almeida D, Pereira R, Coelho-Santos V, et al. Exosomes secreted by cardiomyocytes subjected to ischaemia promote cardiac angiogenesis. Cardiovasc Res. 2017; 113:1338-50.
59. Li F, Truong VX, Fisch $\mathrm{P}$, Levinson C, Glattauer V, Zenobi-Wong M, et al. Cartilage tissue formation through assembly of microgels containing mesenchymal stem cells. Acta Biomater. 2018;10:30409-4.

60. Liu C, Li T, Yang Z, Liu D, Li Y, Zhou Z, et al. Kartogenin enhanced chondrogenesis in cocultures of chondrocytes and bone mesenchymal stem cells. Tissue Eng Part A. 2018;24:990-1000.

61. Mumme M, Barbero A, Miot S, Wixmerten A, Feliciano S, Wolf F, et al. Nasal chondrocyte-based engineered autologous cartilage tissue for repair of articular cartilage defects: an observational first-in-human trial. Lancet. 2016; 388:1985-94.

62. Bhattacharjee M, Coburn J, Centola M, Murab S, Barbero A, Kaplan DL, et al. Tissue engineering strategies to study cartilage development, degeneration and regeneration. Adv Drug Deliv Rev. 2015;84:107-22

63. El Andaloussi S, Mäger I, Breakefield XO, Wood MJA. Extracellular vesicles: biology and emerging therapeutic opportunities. Nat Rev Drug Discov. 2013;12:347-57.

64. Jonitz A, Lochner K, Tischer T, Hansmann D, Bader R. TGF-beta1 and IGF-1 influence the re-differentiation capacity of human chondrocytes in 3D pellet cultures in relation to different oxygen concentrations. Int J Mol Med. 2012; 30:666-72.

65. Lee $\mathrm{SH}$, Che $\mathrm{X}$, Jeong JH, Choi JY, Lee YJ, Lee $\mathrm{YH}$, et al. Runx2 protein stabilizes hypoxia-inducible factor-1alpha through competition with von Hippel-Lindau protein ( $\mathrm{pVHL}$ ) and stimulates angiogenesis in growth plate hypertrophic chondrocytes. J Biol Chem. 2012;287:14760-71.

66. Leijten J, Georgi N, Moreira Teixeira L, van Blitterswijk CA, Post JN, et al. Metabolic programming of mesenchymal stromal cells by oxygen tension directs chondrogenic cell fate. PNAS. 2014;111:13954-9.

\section{Ready to submit your research? Choose BMC and benefit from:}

- fast, convenient online submission

- thorough peer review by experienced researchers in your field

- rapid publication on acceptance

- support for research data, including large and complex data types

- gold Open Access which fosters wider collaboration and increased citations

- maximum visibility for your research: over $100 \mathrm{M}$ website views per year

At BMC, research is always in progress.

Learn more biomedcentral.com/submissions 